\title{
Improved methods for thin, surface boundary layer investigations
}

\author{
H. J. Lin, M. Perlin
}

\begin{abstract}
New techniques are developed to improve the velocity flow-field measurement capability within a freesurface boundary layer region on which progressive capillarygravity waves are present. Due to the extremely thin but rather vortical characteristics of the aforementioned boundary layer, conventional particle image velocimetry (PIV) methods fail to estimate velocity (and vorticity) vectors at an acceptable detection rate. This failure is a direct consequence of optimal PIV parameters that are difficult to achieve in practice for such flow situations. A new technique, Sub-pattern PIV, is developed. This method has features similar to both the super-resolution PIV (Keane et al. 1995) and the particle image distortion (PID) technique (Huang et al. 1993), but is predicated upon a very differential philosophy. Another difficulty that arises in experiments to investigate surface boundary layer flows is that the oscillating and deforming air-water interface has a mirror-like behavior that affects the images, and generates very noisy data. An alternative experimental setup that utilizes the Brewster angle phenomenon is adopted and the specular effects of the free-surface are removed successfully. This Brewster angle imaging, along with the Sub-pattern PIV technique, is used for the target application - a free-surface boundary layer investigation. It proved to be very effective. The methodology of both techniques is discussed, and the modified PIV procedure is validated by numerical probabilistic simulations. Application to the capillary-gravity wave boundary layer is presented in a subsequent paper.
\end{abstract}

Received: 31 July 1997/Accepted: 4 February 1998

H. J. Lin

Department of Mechanical Engineering and Applied Mechanics University of Michigan

Ann Arbor, MI 48109-2125, USA

M. Perlin

Department of Naval Architecture and Marine Engineering

University of Michigan

Ann Arbor, MI 48109-2145, USA

Correspondence to: M. Perlin

This research was supported by the Office of Naval Research Under the University Research Initiative Ocean Surface Processes and Remote Sensing at the University of Michigan, contract number N00014-92-J1650 .
1

\section{Introduction}

In many investigations of flow beneath free-surface waves, a laser sheet oriented perpendicular to the wave fronts is generated to illuminate the spatial field. Light scattered from microseed particles is captured by photographic, CCD, or other recording techniques. By controlling the light source and the imaging system, recorded particle information can be used for PIV or other velocity estimation techniques. Usually, the imaging system is set with a horizontal view angle (Fig. 1a) with its optical axis (lens) set slightly below the quiescent water surface. The image, other than the (desired) light scattered directly from particles, also includes light reflected once from the air-water interface. When the surface is calm or fairly smooth (i.e. short waves are not present), this feature does not cause serious problems since (direct and reflected) images are essentially symmetric to both sides of the interface, and the location of the surface remains detectable. However, if the surface oscillates due to the presence of short capillary waves (wavelength on the order of $\mathrm{mm}$ ), the free-surface becomes difficult to detect. By altering the view angle, the surface reflections can be removed completely. An analysis of the optimum view angle and other parameters are discussed in the next section.

Among existing flow velocity estimation methods, the PIV technique has drawn great attention recently because of its reputation for efficiency and accuracy. Many investigations of PIV schemes have been conducted to improve performance. There exist two major categories of PIV methods - Young's fringe (see e.g. Adrian 1991) and Digital PIV (see e.g. Willert and Gharib 1991). The Young's fringe method utilizes the diffraction and interference phenomena of optical waves, and is usually applied directly to the photographic film. This approach takes advantage of the tremendous resolution that photographic film provides. However, difficulties in capturing time series data render this method laborious and difficult for unsteady flow measurement. On the other hand, the digital PIV approaches utilize rapidly evolving CCD technology, and are widely used in unsteady flow measurements. Using standard $30 \mathrm{~Hz}$ imagers result in a relatively long time interval between images (without using double-exposure techniques). Alternatively, frame-transfer CCD imagers are capable of microsecond time intervals and this restriction is essentially nonexistent. However, limited resolution remains a disadvantage of CCD technology.

For both Young's fringe and Digital PIV approaches, choosing appropriate PIV parameters are critical for a 


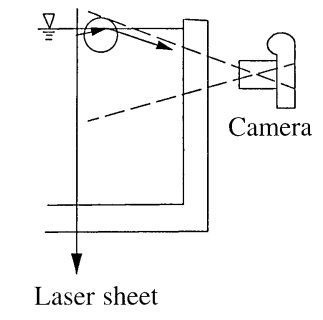

$\mathbf{a}$

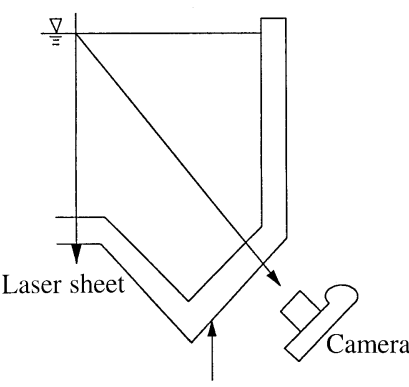

Tilted observation window

Fig. 1a-d. Various setups for imaging the surface along with the subsurface flow. a A conventional imaging system with a horizontal view angle. $\mathbf{b}$ Tilting the camera to the air-water Brewster angle removes most of the reflection from the free surface; however, the glass side-wall still causes imaging difficulties. Two possible solutions: c A tilted observation window. $\mathbf{d}$ A prism attached/sealed on the glass side-wall

successful measurement (Keane and Adrian 1990). That is, the optimal particle size, interrogation window size, time interval, and other parameters have to be determined and applied according to particular flow conditions. For the target application of the present work, a thin boundary layer that features highly vortical flow beneath mechanically generated capillarygravity waves is investigated. Digital PIV is used to measure this unsteady flow. Since an oscillating free-surface is present, a single-exposure method is preferred to facilitate accurate free-surface detection. Because conventional PIV methods were found incapable of measuring these thin boundary layers, the final goal is a procedure that generates velocity estimates with resolution much finer than $1 \mathrm{~mm}$, and is capable of measuring large velocity gradients. The super-resolution PIV technique (Keane and Adrian 1995) and the particle image distortion (PID) technique (Huang et al. 1993) provide a starting point; however, further modification of these techniques in both algorithm and philosophy are required to facilitate the capillary-gravity wave investigation. Hence, a new PIV method, Sub-pattern PIV, is developed. It is analyzed and validated by numerical probabilistic simulations with computer-generated particle images. For a complete discussion of the two experimental techniques along with applications, see Lin (1997).

2

Brewster angle and its application in near free-surface flow investigation

The Brewster angle phenomenon is described first in this section. In general, light can be treated as a transverse electromagnetic wave, with the orientation of the electric field defined as its polarization. Two orthogonal components, $P$ and $S$, are used to describe the polarization. The Fresnel equations, Eqs. (1) and (2), describe the effects of an electromagnetic plane wave incident on the interface between two different dielectric media. These equations relate the ratio of reflected (and trnasmitted) field amplitudes to the incident amplitude by way of the angle of incidence, $\theta_{i}$, and angle of transmission, $\theta_{t}$. The equations are

$R_{p}=\left(\frac{\tan \left(\theta_{i}-\theta_{t}\right)}{\tan \left(\theta_{i}+\theta_{t}\right)}\right)^{2}$

$R_{s}=\left(\frac{\sin \left(\theta_{i}-\theta_{t}\right)}{\sin \left(\theta_{i}+\theta_{t}\right)}\right)^{2}$

By using Snell's law, namely $n_{i} \sin \theta_{i}=n_{t} \sin \theta_{t}$, with $n_{i}$ and $n_{t}$ as the indices of refraction with approximate values of 1.0 for air, 1.3 for water, 1.5 for glass, one can calculate the values of $R_{p}$ and $R_{s}$ for the water-air interface (Fig. 2).

There exist some interesting characteristics of these curves. First, $R_{s}$ and $R_{p}$ both rise to unity at the so-called critical angle (i.e. the angle of total internal reflection). In addition, $R_{s}$ is never zero but $R_{p}$ reaches zero in each case. A specific name, "the Brewster angle or the polarization angle", is given to the angle for which $R_{p}$ equals zero. For angles larger than the critical incidence angles, light is totally reflected. At the Brewster angle, P-polarization of the reflected light vanishes while the S-polarization remains low ( $10 \%$ or less). Thus, most energy is transmitted through the interface.

Usually a laboratory observation system used to measure the velocity field close to an air-sea interface involves three (optical) interfaces: "water-to-air", "water-to-glass", and "glass-to-air". One would like to reduce reflections from the water-to-air interface, that are totally undesirable, and maximize the light passing through the water-glass and the glass-air interfaces (to the imaging system located beyond the glass-wall). A camera tilted upward $42^{\circ}$ measured from a vertical axis, which is the Brewster angle on the water-to-air interface, removes reflection from the free-surface; however, this also produces an angle greater than the critical angle on the glass side-wall, and eliminates all transmitted light

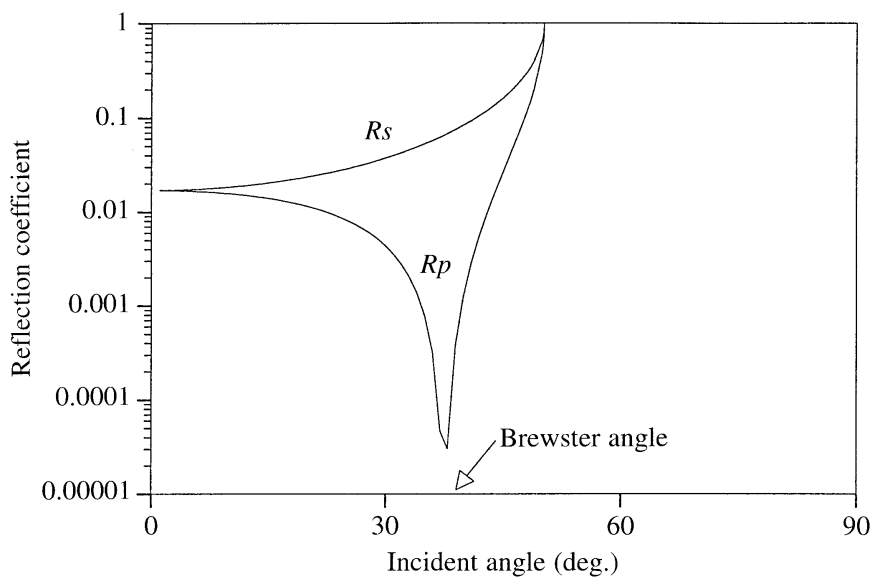

Fig. 2. The reflection coefficients through a water-air interface with $\mathrm{P}$ and $\mathrm{S}$ polarization 


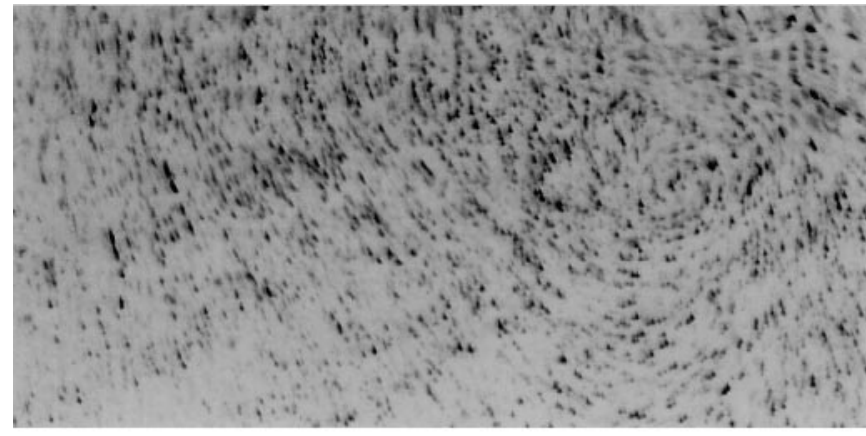

a

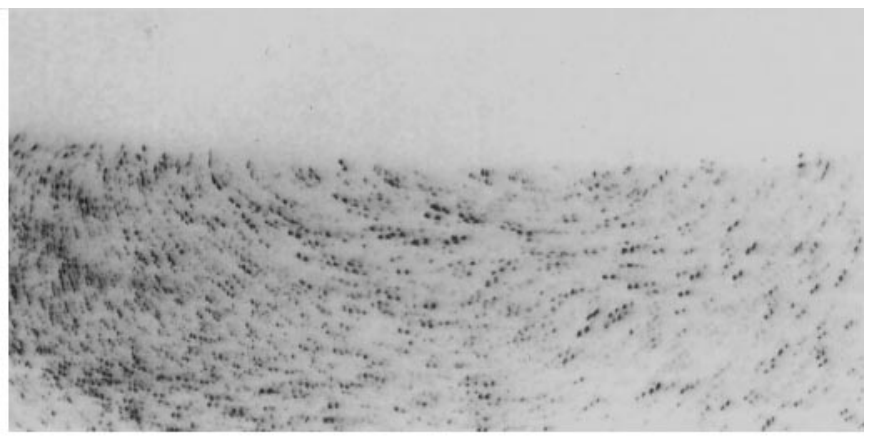

b

Fig. 3. Typical recorded images. On the left, an image recorded without the benefit of viewing at the Brewster angle. The image on the right displays the effectiveness of the Brewster-angle technique in removing unwanted surface reflection of scattered light; hence, straightforward identification of the free surface

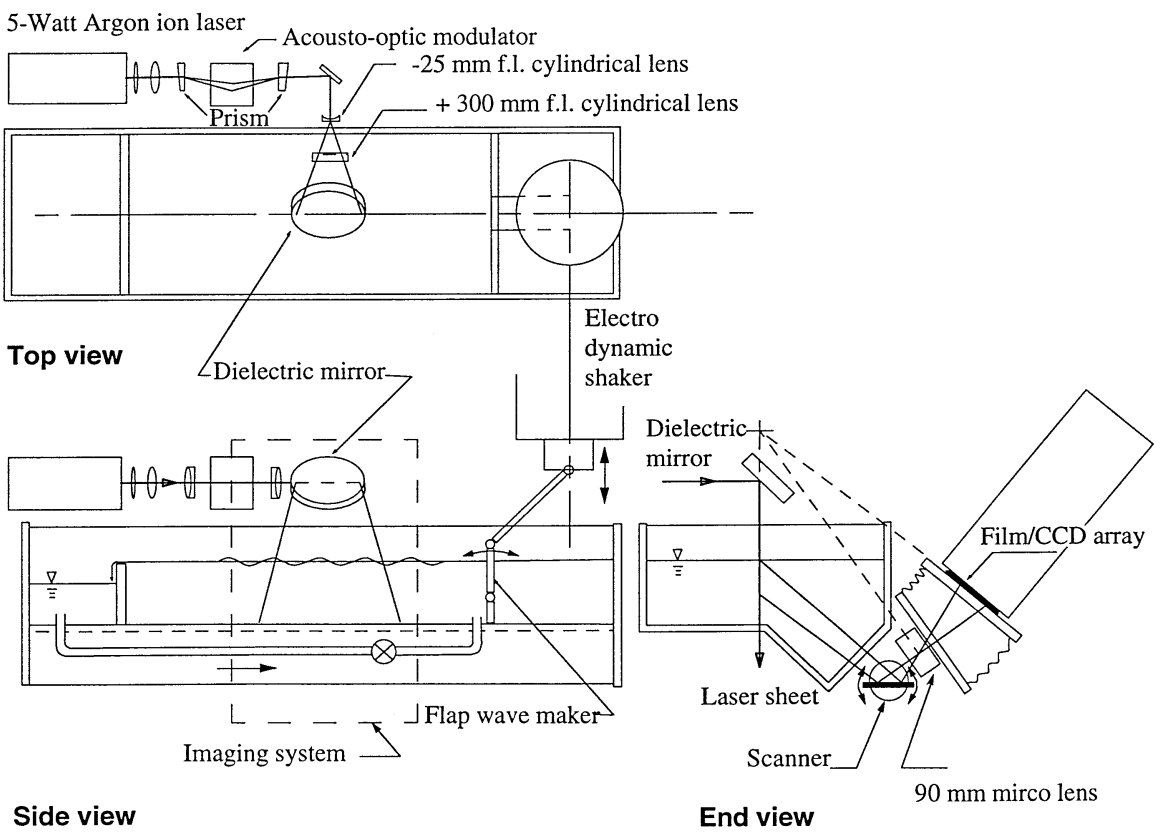

Fig. 4. The experimental setup for the free surface boundary layer investigation (indicated by the circle in Fig. 1b) that makes observation impossible (i.e. no light is transmitted through the glass wall). An inclined observation window corrects this deficiency. A special water channel that has this configuration (Fig. 1c) was constructed to study deep-water capillary-gravity waves. (A complete schematic of the experimental apparatus and channel are shown in Fig. 4).

It should be mentioned that this imaging setup may generate some distortion. That is, the upper portion of the image may have larger magnification than the lower portion of the image. However, it is theoretically a linear distortion and can be corrected easily. The actual distortion depends on the tilt angles of the lens axis and the film/ imaging sensor, and it becomes vanishingly small if the axis of the lens is horizontal and the film/imaging sensor is vertical, which is impractical here. A high-quality lens designed especially for a view camera does decrease the possible distortion.

In Fig. 3, a comparison is presented to demonstrate the effectiveness of the Brewster-angle viewing technique. To produce the left image, Fig. 3a, the camera is positioned as shown in Fig. 1a. It is clear that the free surface is difficult to distinguish. To generate the image shown on the right, Fig. 3b, the setup is as shown in Fig. 1c, and in this image it is straightforward to detect the interface. (In both figures, the film resolution has been lost through digitization and printing; hence, individual particles, and even individual particle pairs, are not always distinguishable.) The technique performs well and functions consistently at the level demonstrated by Fig. 3b. Application to the capillary-gravity wave boundary layer using this technique as well as the Sub-pattern PIV technique is presented in a subsequent paper. 
3

\section{Principles of PIV techniques}

3.0

\section{General definitions}

General principles of PIV methods are presented in this section. Some acronyms and definitions used throughout are presented directly.

Once a flow is seeded with particles and is illuminated, particulate reflectors cast an image on the recording medium (film or imaging sensor). "Optical flow" is defined as the movement of these particle images on the two-dimensional film or sensor plane, whether the associated actual fluid flow is two-dimensional (2-D) or three-dimensional (3-D). Herein, all analyses are based on 2-D optical flow. The magnification and distortion introduced by the imaging optics are not included in the analyses, and the velocity is the optical flow velocity unless otherwise mentioned. Since the target application is 2 -D capillary-gravity waves, the relation between the 2-D optical flow and the actual flow is a simple transformation. A 3-D implementation of the present technique is under development.

\section{1}

\section{Cross-correlation method}

Herein, only cross-correlation methods based on sequential single-exposure images are discussed. However, extension to the multiple-exposure approach can be accomplished without significant modification.

In the cross-correlation method, two sequential frames of particle images are required for the displacement estimate. The cross-correlation coefficient function is defined as

$R(\xi, \zeta)=\frac{\int_{A} I(x, y) S(x-\xi, y-\zeta) \mathrm{d} A}{\sqrt{\int_{A} I^{2}(x, y) \mathrm{d} A \int_{A} S^{2}(x-\xi, y-\zeta) \mathrm{d} A}}$

where $A$ is the area of interrogation window, $I$ and $S$ are intensity distribution functions of the first image and the second image, respectively. Particle images bounded by a specific border form a particle image pattern (PIP). Some special PIP's are defined now (Fig. 5). The particle images within a chosen interrogation window, that is described by $A$ in Eq. (3), of the first image form the interrogation particle image pattern (I-PIP); the same group of particles forms a slightly different pattern on the second image, and is called the related particle image pattern, R-PIP. The arbitrary vector $(\xi, \zeta)$ imposes a shift on the integration area and is bounded by the so-called searching range, chosen to be at least the magnitude of the maximum expected particle displacement. The PIP that occupies the interrogation window on the second image (shown as the dashed-line square in Fig. 5), extended by the searching range on each of the four sides, is called the search particle image pattern (S-PIP). A window that has the same size and shape as the I-PIP, but is shifted by the arbitrary vector $(\xi, \zeta)$ is called a search window, and is denoted by $\operatorname{SW}(\xi, \zeta)$. There exists a particular search window, namely $\operatorname{SW}\left(\xi_{0}, \zeta_{0}\right)$, that has coincident center with the true R-PIP. And this particular displacement vector $\left(\xi_{0}, \zeta_{0}\right)$ is the desired output of the PIV algorithm. $R(\xi, \zeta)$, the value of the cross-correlation function at point $(\xi, \zeta)$, is essentially the correlation integral of

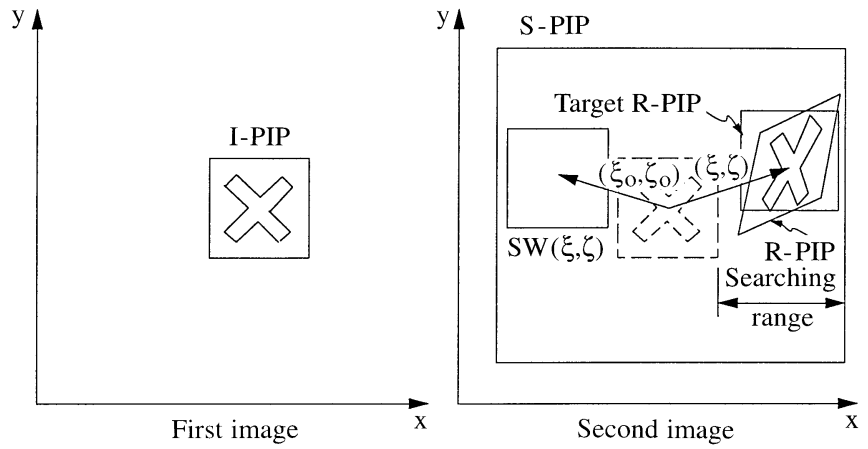

Fig. 5. A schematic representation of I-PIP, S-PIP, R-PIP, and target R-PIP

the I-PIP and the PIP bounded by $S W(\xi, \zeta)$, and it is an indication of the similarity between these two PIP's. According to the fundamental assumption that the I-PIP and the R-PIP contain similar particle image pattern characteristics, and the fact that $S W\left(\xi_{0}, \zeta_{0}\right)$ and the R-PIP have a coincident center, the magnitude of the correlation integral equivalently indicates the likelihood that $(\xi, \zeta)$ is the target displacement vector $\left(\xi_{0}, \zeta_{0}\right)$.

It can be shown that the cross-correlation function has a theoretical maximum value of one, and that it occurs only if an identical pair of R-PIP and I-PIP can be found. In an actual flow, differences between the I-PIP and the R-PIP usually exist due to particles moving out-of-plane, blocked particles (i.e. particles remain in the illuminated volume but are obscured by foreground particles), and velocity gradient effects. These three factors are responsible for non-correlated particles that affect the pattern characteristics. In addition, velocity gradient effects may also significantly change the shape of the R-PIP as compared to the I-PIP. (In Fig. 5, the R-PIP is presented as a rhombus, but in general, it can be a distorted, curved figure.)

In a cross-correlation PIV method, the apparent signal is defined when the cross-correlation coefficient is the global maximum of the cross-correlation function,

$R\left(\xi_{0}, \zeta_{0}\right)=\operatorname{Max}\{R(\xi, \zeta)\}$

and the apparent signal is assumed to represent the displacement of the related images. Other local maxima are usually referred to as cross-correlation noise, and they are large cross-correlation integrals due to randomly distributed, nonrelated particle images.

\section{2}

\section{Accuracy, resolution, detection rate and validity of PIV methods}

Since the assumption that the apparent signals represent the displacements does not mathematically guarantee a correct (or sufficiently close) estimate, the PIV method can be classified as a Monte Carlo algorithm. This raises the question of validity found frequently in most PIV methods - spurious velocity vectors are realized in the generated velocity fields. A good PIV algorithm, however, provides a high confidence level that correct estimates are generated. There are two common methods used to decide whether an estimate is a valid estimate. First, a signal-to-noise (S/N) threshold is chosen and the ratio 
between the apparent signal and the (apparent) noise peak (the second largest cross-correlation coefficient) must be greater than this prescribed threshold or the data discarded (Adrian 1991). A second method compares an estimate and the median of its neighboring estimates. Once again, a threshold is chosen and used to determine whether an estimate is valid (Westerweel 1994). The detection rate is the percentage of accepted estimates. However, the detection rate does not necessarily reflect the validity, defined by the percentage of occurrence at which the apparent signal is sufficiently close to the true signal (i.e. noise is not mistaken as a signal). The validity is a better measure of the confidence level of a PIV algorithm. However, this value is indeterminate since the true velocity is unknown. Hence, a numerical probabilistic simulation is adopted usually to validate a PIV algorithm. In addition to the validity, the accuracy of a PIV scheme is determined by how precisely the signal can be located, and it is dictated mostly by the resolution of the imaging system, even when a sub-pixel interpolation technique is applied. As the velocity is estimated by dividing the displacement vector by the time interval, using a shorter time interval results in a lower relative accuracy due to the fixed accuracy in displacement estimation (Boillot and Prasad 1996). Therefore a sufficient time interval must be retained for a fixed velocity. Since only one approximate displacement results from each successful search process, the resolution of a PIV method is determined by the number of velocity vectors that can be extracted from the entire image, and it is related directly to the size of the interrogation window.

\section{3}

\section{PIV parameters and their effects}

To increase the validity of a PIV method, a set of optimal PIV parameters (i.e. seeding particle size, seeding density, interrogation window size, recording time interval, etc.) has to be chosen. A systematic study of the optimal PIV parameters for different velocity and velocity gradient distributions has been presented by Keane and Adrian (1990) and Keane et al. (1995).

According to Adrian, the following criteria are necessary to optimize the interrogation of a general velocity field by correlation analysis. First, to ensure sufficient particle image pattern distinguishability,

$D^{2}\left(\Delta z_{0}\right) C=N>N^{*}$

must be satisfied. $D$ is the size of the interrogation window, $\Delta z_{0}$ is the laser sheet thickness, $C$ is the particle concentration defined as number of particles/unit volume. Thus, $N$ is the number of particles within the interrogation window, and $N^{*}$ is the required minimum number of particles within a window. Adrian has suggested that $N$ be at least 12 to ensure a successful estimate. In general, a larger $N$ results in a better particle pattern distinguishability, or a higher tolerance to uncorrelated particles, as the magnitude of $R\left(\xi_{0}, \zeta_{0}\right)$ is in direct proportion to the number of displaced (but related) image pairs remaining. Nevertheless, having more particles within an interrogation window usually increases the probability that the center of gravity of the PIP is close to the geometric center of the interrogation window (i.e. a lower detection bias), such that the estimate velocity is a more accurate representation of the actual velocity.

The second restriction arises from velocity gradient effects. For optical flows, the criterion is

$\left|\frac{\partial \mathbf{u}}{\partial x}\right| \Delta t<\frac{d}{D}$

$\Delta t$ is the time interval between images, $d$ is the average diameter of the particle images, and $|\partial u / \partial x|$ is a general expression for the maximum velocity gradient (of the optical flow) that exists in the plane of the laser sheet. (Note that the magnification is set to one here as in Adrian's criterion, as the analysis for the present paper is based on the optical flow.)

The third effect, particles moving out-of-plane, includes two criteria,

$\Delta t<\frac{\Delta z_{0}}{4 u_{z}}$

and

$\left|\frac{\partial \mathbf{u}}{\partial z}\right| \Delta t<\frac{d}{M} \frac{1}{\left(\Delta z_{0}\right)}$

$u_{z}$ is the maximum expected (actual) velocity component in the direction perpendicular to the laser sheet, $M$ is the magnification, and $|\partial \mathbf{u} / \partial z|$ is the maximum expected (actual) velocity gradient in this direction. The criteria, Eqs. (7) and (8), are usually less restrictive if the laser sheet is arranged parallel to the dominant velocity components. Thus, the laser sheet thickness can usually be adjusted to match the depth-of-field of the imaging system.

There is also a one-quarter rule, namely $D / 4>|\mathbf{u}|_{\text {mean }} \Delta t$, suggested for auto-correlation methods to ensure that a sufficient portion of the particles remain inside the interrogation spot. Here, $|\mathbf{u}|_{\text {mean }}$ is the average magnitude of the velocity. If one is using cross-correlation methods with un-equally sized I-PIP and S-PIP, or is using a similar PIP size arrangement with double-exposure, single-frame image data, this requirement can be discarded as it is here. However, it has to be satisfied when the Young's fringe method is applied since a single interrogation laser beam cannot perform an un-equally sized cross-correlation integration.

\section{4}

\section{The particle image distortion technique and super-resolution} PIV

There are various PIV methods that improve performance; specifically, they improve the accuracy and resolution. Two recently published techniques that relate directly to the new Sub-pattern PIV method are described briefly.

The particle image distribution (PID) technique, as modified from conventional PIV, was presented by Huang et al. (1993). This method is essentially an iterative PIV technique with consideration of the particle image patterns' distortion. The velocity field acquired from the previous iteration is used to generate an artificial, locally linear distortion of the I-PIP, and these altered I-PIP's are used in subsequent PIV. This procedure is performed iteratively, until a converged velocity estimation is achieved. 
This method is intended to improve the accuracy of conventional PIV when velocity gradients are present. However, as no velocity estimations are made initially, only a conventional PIV scheme with non-distorted I-PIP's can be adopted for the first approximation. This first approximation is restricted somewhat to the same PIV parameters as the conventional PIV approach. That is, the restriction of the maximum allowable velocity gradient is retained, and subsequent approximations cannot be performed without a valid (first) velocity field estimation. Huang et al. (1993) do discuss a process by which bad data are corrected by smoothing; however, in the immediate vicinity of the free surface, the authors had difficulties obtaining a valid correction using their technique. The initial estimated displacements must be close to the desired targets for the subsequent approximations to converge to a more accurate estimate; otherwise, the true signal might decrease further and still generate invalid data.

A method developed by Perkins and Hunt (1989) uses the velocity field from cross-correlations of large interrogation spots to guide the individual particle image displacement estimates. In a recent paper by Keane et al. (1995), super-resolution PIV, a similar method with subinterrogation-spot PIV, is presented. It applies conventional PIV to the interrogation spot with optimal diameter, and then several smaller spots within are chosen from which to determine individual displacements similar to the particle tracking technique. As with the PID described previously, the restrictions in choosing PIV parameters for this method remain the same as those of a conventional PIV method. However, a higher in-plane resolution is achieved by this technique.

\section{4}

\section{Modification behind Sub-pattern PIV - difficulties in} choosing PIV parameters

In the previous discussion of PIV parameters, one must be aware that Eq. (5) favors a large interrogation window; Eq. (6) suggests the opposite. Other parameters, recording time interval, seeding density, and seeding particles, must be adjusted carefully to satisfy both requirements. However, it is frequently found that the optimal condition is very difficult (or impossible) to achieve, especially when the flow has large velocity gradients and widely distributed velocity scales (i.e. turbulent flow), or when the investigated area is extremely small.

Using Eq. (6), a higher $d / D$ ratio (i.e. larger particles or the same particles with higher seeding density and smaller interrogation window) ensures the validity of the PIV estimation by overcoming the velocity gradient effects. However, larger particles may not correctly follow the motion of the water, and larger particle images may also decrease the accuracy in locating the signal; a smaller window with higher seeding density may be difficult to achieve. The light sources are, in most PIV applications, ion lasers with wavelengths in the range of microns; thus a particle image cannot be less than the order of $10 \mu \mathrm{m}$ due to optical diffraction regardless of its actual size. Also, finer and finer particles eventually result in a colloidal-like suspension that is clearly undesirable. This restricts using finer interrogation windows as overlapped particle images are undesirable. Thus, the flexibility to choose a proper $d / D$ ratio is inhibited.

Decreasing the time interval between images usually improves the flexibility of choosing PIV parameters; however, the time interval is often restricted by the imaging system, and it also decreases the relative accuracy of the velocity estimations since the estimated velocities are calculated by the displacements divided by the time interval. The accuracy of the displacement estimation is limited by the fixed pixel-resolution of the imaging system. A sufficient time interval ensures a (relatively) accurate velocity estimation.

The present objective is to apply a new, Sub-pattern PIV measurement technique to determine the flow field beneath capillary-gravity water waves. It is believed that, beneath capillary-gravity waves, the flow is essentially irrotational (with low velocity gradients) except in a very thin layer immediately beneath the free-surface. Using conventional PIV methods to determine the velocity/vorticity field does not produce reliable results as the window size must be (one order) smaller than the length scale of this thin region (boundary layer), and the aforementioned limitations make this very difficult (impossible) to achieve. Also, due to interactions between seeding particles and the free-surface, the seeding must remain at a relatively low density. (It was found during drift-current velocity measurements, that if the seed density at the surface exceeded a threshold, the particles interacted with each other and the surface and formed a "matt-like structure" that somewhat resisted the wave motion, especially with frequencies of about $16 \mathrm{~Hz}$. Uncoated hollow glass spheres were less influenced by this phenomenon and thus were used.) With this restriction, the necessarily small interrogation window contains insufficient particles to assure a valid estimation of the flow field. Super-resolution PIV may be conducted using a larger interrogation window to satisfy Eq. (5) as the first approximation, then a decreased window size for the second estimation to achieve finer resolution. This approach is problematic as the velocity gradient is larger than allowed (i.e. Eq. (6) cannot be satisfied). Since velocity estimates are absent above the free-surface, and most spurious velocity estimates occur at the upper-most grid points (the highly vortical boundary layer region), correcting invalid data by comparison and interpolation is not a valid option. Likewise, the authors had difficulties using the PID technique as unrecoverable data occur on the boundary for the first approximation (i.e. the first iteration).

An effective PIV scheme for measuring extremely small boundary regions is required. It should maintain large interrogation windows and a sufficient time interval, but avoid invalid data caused by velocity gradient effects. That is, the restriction in choosing PIV parameters due to velocity gradient effects must be relaxed through the use of an alternate procedure. This can be accomplished by utilizing two different-sized interrogation windows, each window satisfying either Eq. (5) or Eq. (6), but not both criteria simultaneously. A modified PIV method is developed and now discussed that utilizes information extracted from each window.

As described in the previous section, the difficulty in applying appropriate PIV parameters limits the application of conventional and existing PIV methods to flows with small velocity gradients. The proposed Sub-pattern PIV facilitates 
large velocity gradients in very thin boundary layers without decreasing the time interval by including sub-interrogation windows that are smaller than the original interrogation windows. This concept is similar to that of super resolution $\mathrm{PIV}$ as it uses conventional PIV and subsequent sub-interrogation-spot PIV/PTV. However, it is founded upon a different philosophy as regards Eqs. (5) and (6). With two differentsized interrogation windows, Eq. (5) is satisfied by the full-sized interrogation window - it is large enough to include a sufficient number of particles (to ensure distinguishable PIP characteristics); and Eq. (6) is satisfied by the size of the sub-interrogation window - it is small enough to facilitate and eventually compensate for the velocity gradient. As the sub-interrogation window does not include sufficient particles to form a distinguishable particle image pattern, it is referred to as a "sub-pattern" to differentiate it from the fullsized interrogation particle image pattern. Since neither the full-sized nor the sub-interrogation window satisfy both Eqs. (5) and (6), a valid PIV estimation cannot be achieved by either of them independently. However, combining their cross-correlation results solves the validity problem. Such an algorithm is developed herein. And by generating an appropriate artificial image distortion (similar to the PID technique), the velocity gradient effects are overcome and Eq. (6) is no longer restrictive. The displacement information determined by the sub-interrogation-window $\mathrm{PIV/PTV} \mathrm{is} \mathrm{sufficient} \mathrm{for} \mathrm{high-spatial} \mathrm{resolution} \mathrm{with} \mathrm{compara-}$ ble sub-pixel accuracy.

\section{5}

\section{Prediction and simulation of cross-correlation signal and} noise

As two different-sized interrogation windows that do not simultaneously satisfy Eqs. (5) and (6) are adopted in the present Sub-pattern PIV, changes in signal and noise are discussed first. Since the two windows may result in very high $d / D$ ratios or very few included particles, an extensive quantitative investigation is necessary. Using a simulation with computer-placed particles and chosen velocity gradient so that the actual (true) initial and final images and velocity vectors are known, a comparison is made with the results from the Sub-pattern PIV technique. That is, a series of tests with randomly-generated particle images simulate the changes in signal and noise that occur when a velocity gradient is present. The results provide validation of the Sub-pattern PIV technique. First, using Poisson statistics, signal decay and noise are investigated.

By assuming that the particle distribution is Poisson, quantitative predictions of the signal decay (Fig. 6) and the maximum possible noise (Fig. 7) are calculated. In the graph of signal decay, the horizontal axis represents the relative velocity gradient; it has a value of one when Eq. (6) is enforced. For any position in the image, if the chosen interrogation window is larger than the one suggested by Eq. (6), the signal cross correlation coefficient will decay. The vertical coordinate of Fig. 6 represents the probability of finding a target crosscorrelation coefficient that is greater than a specified value. It is seen in Fig. 6 that, for example, the probability of finding the signal larger than 0.5 is slightly higher than 0.5 , if the local velocity gradient is twice as large as that suggested by Eq. (6),

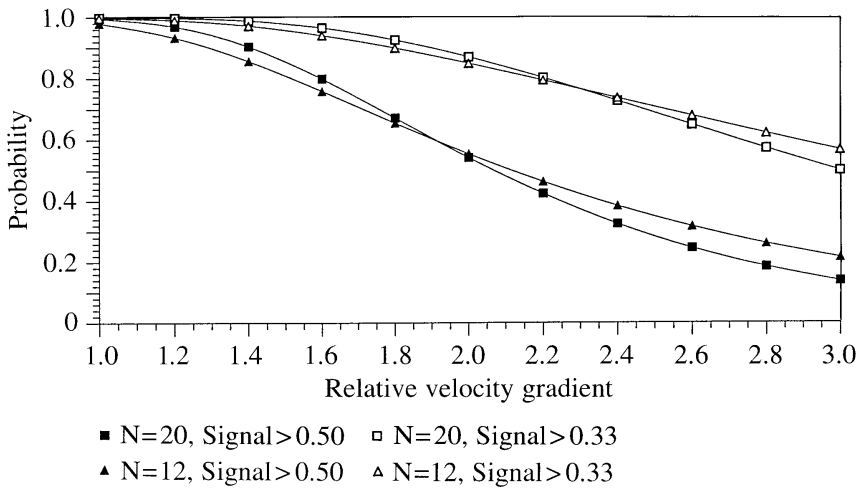

Fig. 6. Signal decay predicted by Poisson statistics. The relative velocity gradient has a value of one when Eq. (6) is enforced. The vertical coordinate indicates the probability that the true signal cross-correlation coefficient is larger than 0.5 and 0.33 for I-PIP's containing different numbers of particles

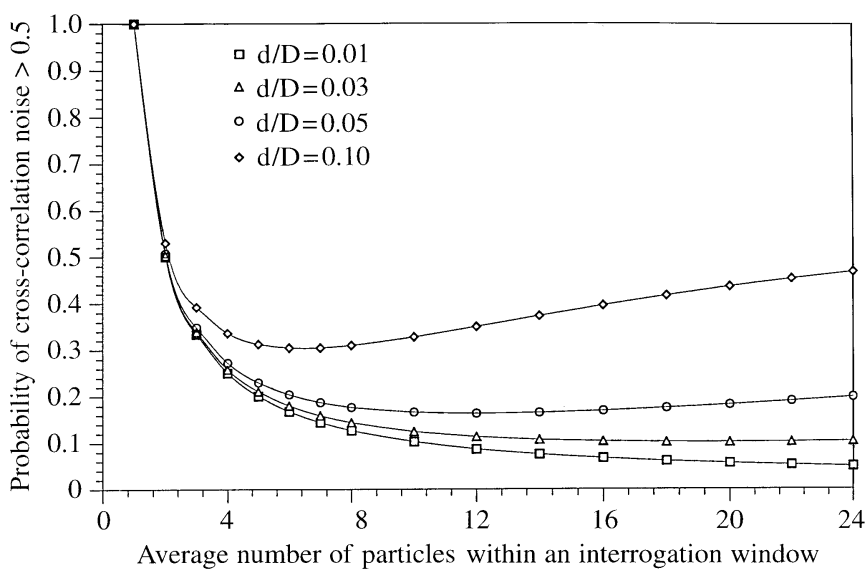

Fig. 7. Cross-correlation noise predicted by numerical probabilistic simulation

and that the particle density (comparing different $N$ ) has only minor effects on signal decay.

The peak noise that causes the validity problem is important. Fig. 7 shows the probability of finding "maximum noise" that exceeds a "particular threshold" with different seeding density and different $d / D$ values. Although only one case of "particular threshold", 0.5 is the value used, is presented in Fig. 7, simulation with other thresholds can be calculated similarly. According to Fig. 7, for an arbitrarily chosen seeding density (average number of particles within an interrogation window), for example 12 particles, the probability of finding "maximum noise" that exceeds 0.5 is about 0.4 for $d / D=0.10$.

Interpreting the results from Figs. 6 and 7 shows a high probability that the "maximum noise" may exceed the decayed signal. For example, if the relative velocity gradient (RVG, defined as $(\partial u / \partial y)(\Delta t) D / d)$ is twice the limit suggested by Eq. (6) (i.e. only half of the interrogation window is effective), there exists only a $30 \%$ chance that the signal may confidently exceed the maximum noise, but there exists about a $30 \%$ chance that the noise is larger than the decayed signal. The other $40 \%$ are uncertain, possibly comparable, signal and noise magnitude. These are unacceptable values. 


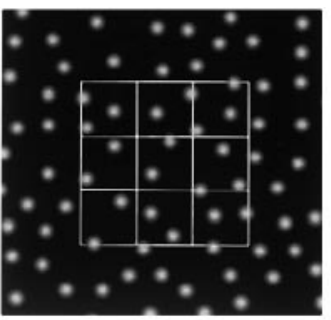

a

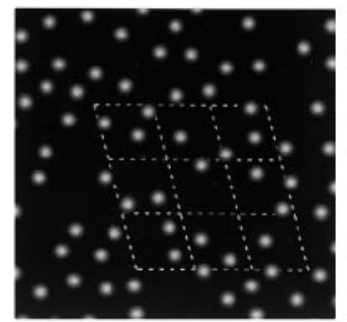

b
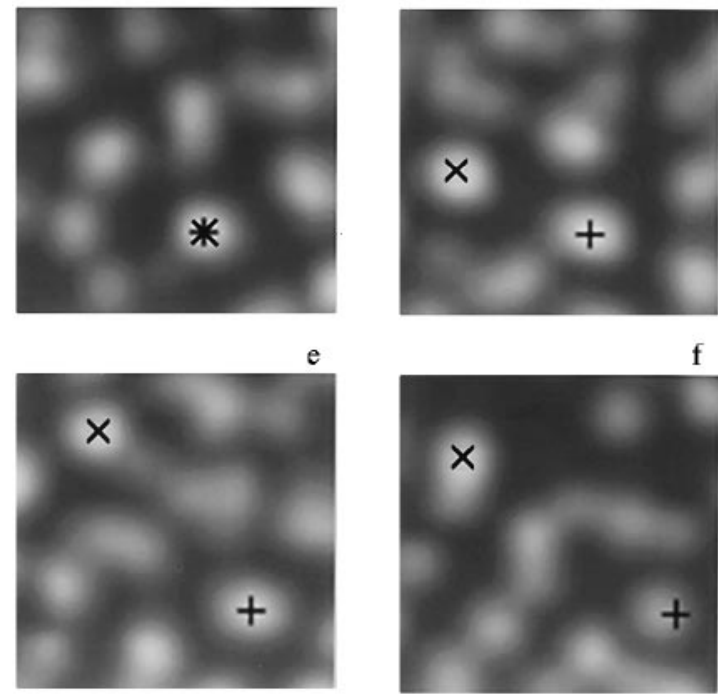

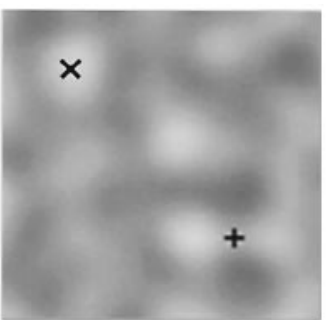

c

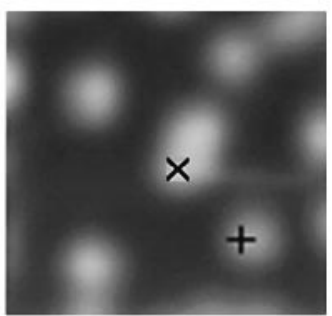

g

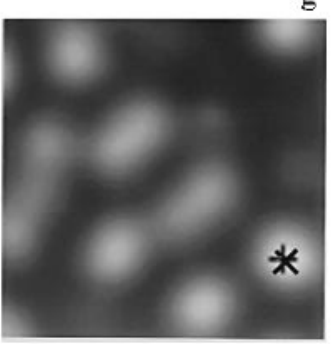

$\mathrm{k}$

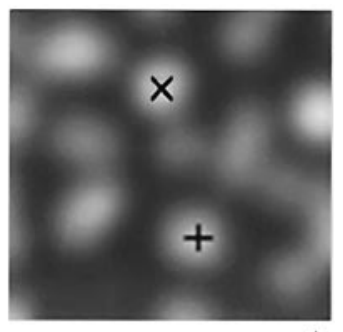

d
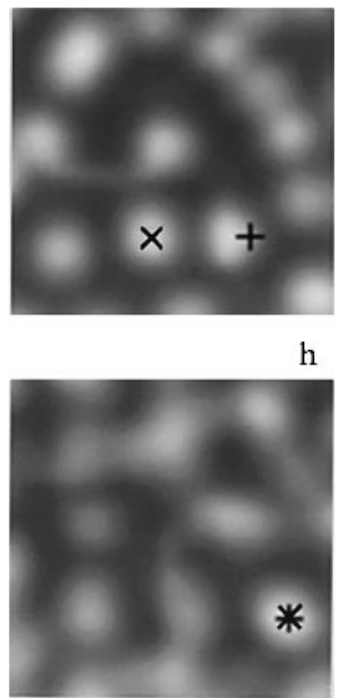

1

Fig. 8a-l. Gray-scale representation of the cross-correlation integral and the full-size interrogation window and sub-interrogation windows. The " + " indicates the true signal and the " $x$ " indicates the apparent signal

Next, a series of tests with randomly-generated particle images simulate the changes in signal and noise that occur when a velocity gradient is present. Fig. 8 shows a typical graphical result. The two images in Figs. $8 \mathrm{a}$ and $\mathrm{b}$ are the computer-generated first and second particle images. This particular image pair represent particle motion with a constant product of the velocity gradient and the time interval, namely $(\partial u / \partial y) \Delta t=0.3$ (also $u \Delta t=v \Delta t=0.07 D)$. The large square in Fig. $8 \mathrm{a}$ and the large region enclosed by dashed lines in Fig. $8 \mathrm{~b}$ indicate the motion of the simulated optical flow. About 20 particles are present in the interrogation window (the largest square within Fig. 8a) with particle size $d / D=0.1$. The image also includes uncorrelated particles. The resulting crosscorrelation is shown in Fig. 8 c, a case with $20 \%$ uncorrelated particles. The velocity gradient is three times greater than the limit suggested by Eq. (6). Fig. $8 \mathrm{c}$ shows the resulting, invalid conventional PIV estimation. (The " + " indicates the true signal and the " $x$ " indicates the apparent signal.) The apparent signal is not even close to the actual (true) target. In the particular case shown in Fig. $8 \mathrm{c}$, the true target is located actually between the third and the fourth peaks of the crosscorrelation, although the top four peaks have comparable values that are close to 0.4 . Figs. $8 \mathrm{~d}-1$ are gray-scale results of cross-correlation integrals of the same images, but the PIP size is chosen as $(1 / 3) D$, shown as the smallest squares in Fig. 8 a. Thus, the velocity gradients in these sub-windows are within the limit specified by Eq. (6); however, the number of particles contained in the interrogation window is less than an appropriate number (i.e. Eq. (5) is not satisfied). The cross-correlation function shows several peaks for each cross-correlation integral, and the true target displacement is always one of them as long as each small interrogation window contains at least one related particle pair. Figs. 8e, $\mathrm{k}$ and 1 exhibit an apparent signal that is very close to the correct location; however, other cross-correlation integrals obviously do not have coincident locations of the target (true) signal and the apparent signal (maximum peak). The signal/noise ratio is approximately one for each of these nine cases. Hence, the peak cross-correlation integral of the sub-windows does not provide definitive information.

In Fig. 9, a series of simulations with two relative particle sizes, two relative velocity gradients, two numbers of particles, and two percentages of uncorrelated particles is presented, six of the sixteen possible combinations. There are more than three-hundred cases simulated for each of the six combinations presented, and computer-generated particle images that satisfy the Poisson distribution are used. As the true particle displacements are known, the (true) target and maximum noise are located. (The maximum noise is defined as the largest cross-correlation coefficient other than the true signal.) Note that if the maximum noise is larger than the true signal, the noise is taken as the apparent signal, and incorrect 


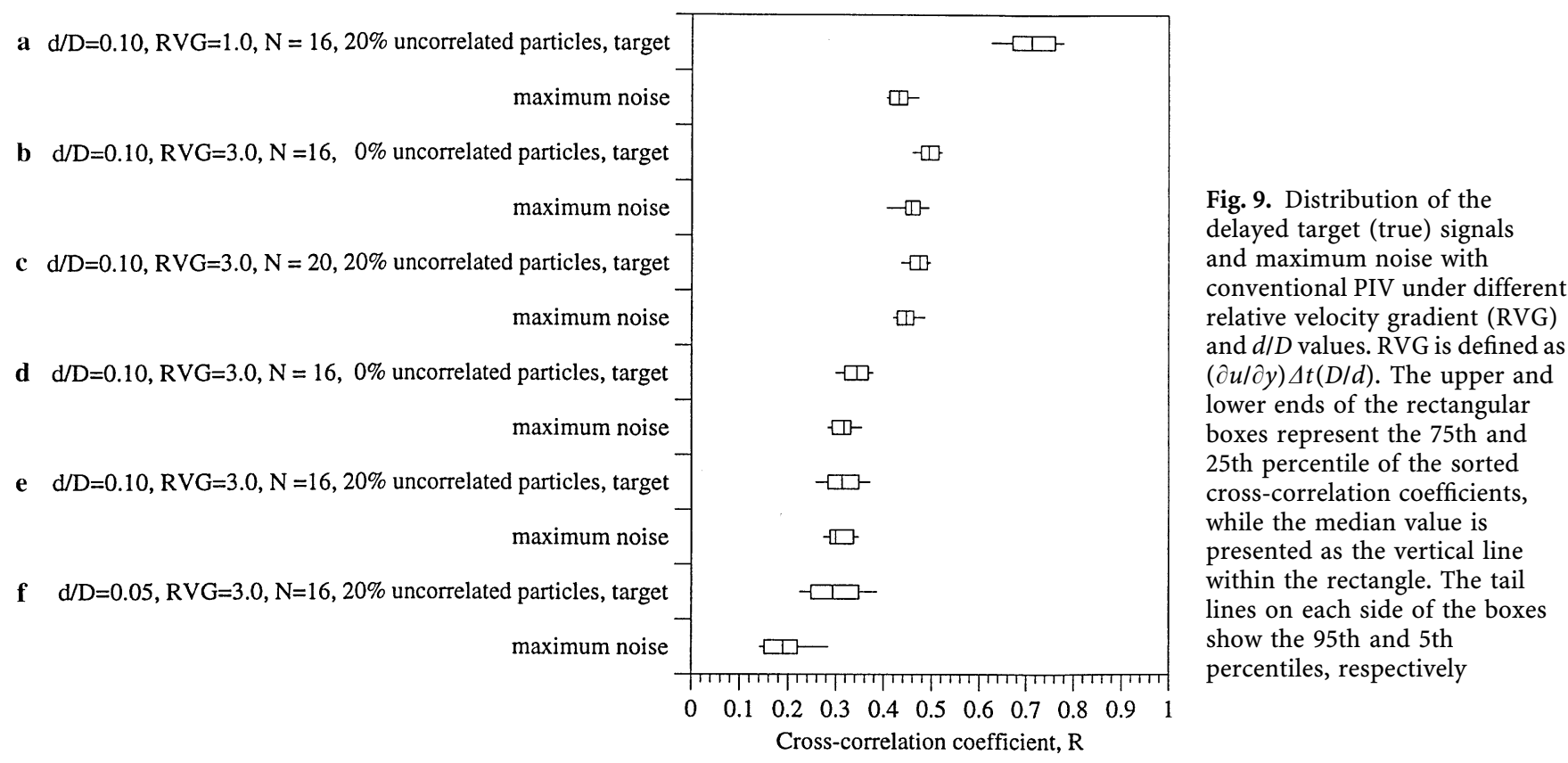

displacement estimates result. In Fig. 9, the magnitudes of cross-correlation coefficients are presented as a distribution. The upper and lower ends of the rectangular boxes represent the 75th and 25th percentile of the sorted cross-correlation coefficients, while the median value is presented as the vertical line within the rectangle. The tail lines on each side of the boxes show the 95th and 5th percentiles, respectively. When the velocity gradient is less than the limit suggested by Eq. (6), simulations represented by Fig. 9a, the distribution of maximum noise and target signal are separated clearly. That is, choosing apparent signals as targets, in general, will not generate invalid estimates. However, when the velocity gradient is well-over the limit, the distributions of the noise maximum and target cross-correlation coefficients overlap. Mistaking noise as the target occurred in $37 \%$ of the cases with $N=16$ and $20 \%$ un-correlated particles, and $10 \%$ of the cases with $N=16$ with no uncontrolled particles. As can be seen in the figure some overlap occurred for the true target and noise in all but simulation (a).

\section{6}

\section{Sub-pattern PIV procedure}

Experience that the optimal parameters are impossible to achieve and the invalid estimation demonstrated above using conventional PIV with non-optimal window size or seeding density indicates clearly that an improved technique is required. Th Sub-pattern PIV procedure circumvents these difficulties and its methodology is presented directly.

Similar to the PID method, an artificial image distortion is included in Sub-pattern PIV. However, unlike PID, conventional PIV is not adopted for the first iteration. As shown previously, conventional PIV yields invalid estimates for velocity gradients larger than the allowable value. To determine the required image distortion (that essentially removes the actual velocity gradient effects that appear in the latter image and thus facilitates use of conventional PIV techniques using the first image and the latter, purposely distorted image), appropriate sub-interrogation windows have to be used. The length of a side of these sub-interrogation windows still has to satisfy Eq. (6), but Eq. (5) is discarded at this point. Here, stated as an empirical result, 1/3 of the length of the full-sized interrogation window gives the best results for images with $d / D$ approximately 0.1 . Since the distribution of individual particle images is not necessarily homogeneous, and the goal at this juncture is to predict a (sufficiently) close velocity gradient value, only the most-concentrated particle image sub-patterns are required. That is, only three sub-interrogation windows properly chosen are required to give a first-approximation to the actual distortion between images. A procedure that applies a convolution integral to the original image using a top-hat function across the interrogation window (i.e. a unit-function with zeroes padded outside the (small or sub-) interrogation window), and then searches for peaks, is sufficient for locating the most-concentrated particle image sub-patterns. The three most-concentrated particle image sub-patterns are chosen as long as each represents independent data. The constraint to ensure that each represents independent data is that the sub-interrogation windows must not exceed $\frac{1}{2}$ overlap on each other.

In Fig. 10, using the same particle patterns presented in Fig. 8 for which it was shown that conventional PIV failed, the step-by-step results of the Sub-pattern PIV procedure is presented. In Fig. 10a, the large white-outlined square is the original full-sized I-PIP, and the smaller squares mostly within are the chosen sub-patterns. Three sub-pattern displacement estimates are sufficient for prediction of locally linear distortions; however, more estimates generate an over-determinate system and stabilize the prediction, and can be used to 

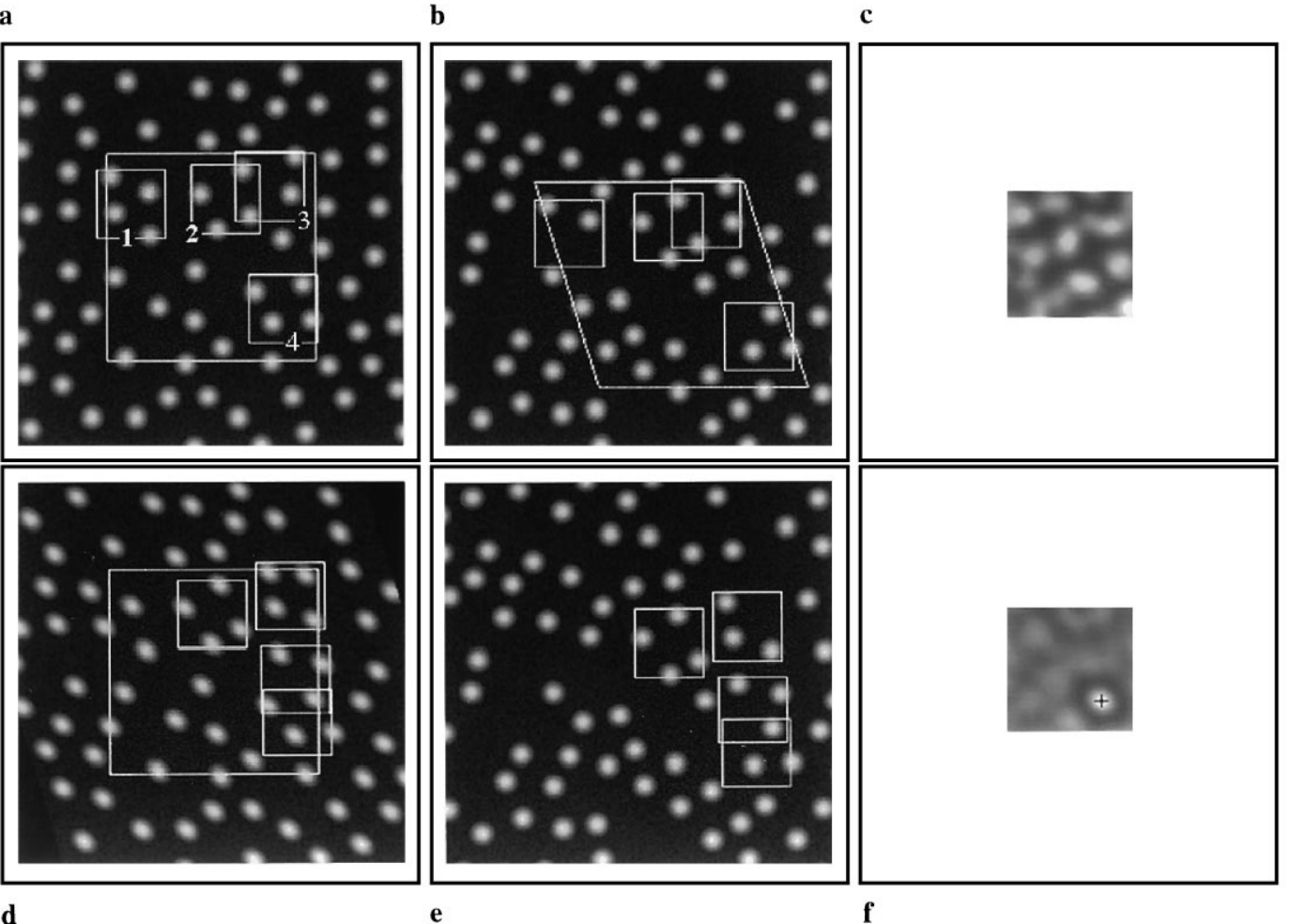

Fig. 10a-f. Step-by-step results of the Sub-pattern PIV procedure. The "+" indicates the location of the true signal

predict a higher-order distortion. Four sub-patterns (the number used in later computer simulations) proved to be appropriate to predict the locally linear distortion and also provide good tolerance to inhomogeneous particle distributions.

The second step in the procedure is performing crosscorrelation integrations between each sub-pattern I-PIP (SubIPIP) and the S-PIP. Fig. 10c shows the result of the crosscorrelation integral between the Sub-IPIP (the small square labelled with a 2 in Fig. 10a) and the S-PIP. Since the maximum possible velocity is bounded by a pre-estimate value (i.e. the search region), the integral result outside the region is discarded. As described previously, these (small) sub-pattern generated signal cross-correlation coefficients are without theoretical decay; however, as each sub-pattern does not contain sufficient particles, the noise is so large that the resulting signal/noise ratio is poor. Choosing the largest cross-correlation coefficient as the target may result in an invalid displacement. (See also Fig. 8.) The only reasonable assumption is that the true displacement is one of these local maxima. Thus, the largest $m$ peaks are stored.

Since the (true) displacement of every sub-pattern can be used to approximate the velocity gradient within the (full-sized) interrogation window, using one peak from each sub-pattern and combining them gives a possible particle image pattern deformation (as caused by the velocity gradient). And since it is unknown which is the correct combination, the largest $m$ peaks from each sub-interrogation window cross-correlation integral are considered. The number, $m$, is interpreted as the (average) number of particles that appear in the S-PIP associated with each sub-interrogation particle image pattern (Sub-IPIP). Its value is determined by

$m=N\left(\frac{D^{\prime}+2 \Delta s_{\max }}{D}\right)^{2}$

$D^{\prime}$ is the length of a side of the sub-interrogation window, $\Delta s_{\max }$ is the searching range defined previously, and $N$ is the average number of particles within the full-sized interrogation window. A total number of possible combinations, $m^{n}$ ( $n$ is the number of sub-interrogation windows), is encountered. This total number may exceed a thousand; however, most can be eliminated by the following rules, essentially based on the pre-estimated upper-bound of the expected velocity gradients, and also on the assumption that the flow is highly incompressible.

First, a maximum velocity-gradient criterion is applied. The data must satisfy the following equation or they are rejected:

$\frac{\left|x_{i}-x_{j}\right|}{\left|\left(x_{i}+\delta x_{i k}\right)-\left(x_{j}+\delta x_{j k}\right)\right|}<\left|\frac{\partial \mathbf{u}}{\partial x}\right| \Delta t, \quad i \neq j$

$x_{i}$ is the location vector of the $i$ th Sub-IPIP center, and $\delta x_{i k}$ is the possible sub-pattern displacement vectors indicated by the $k$ th cross-correlation peak of the $i$ th sub-pattern. $|\partial u / \partial x|$ is the general expression for the maximum velocity gradient in Eq. (6). That is, the distance change between any two sub-patterns should not be larger than the maximum allowable as determined from the velocity gradient. 
Another criterion, mathematically described as

$$
\begin{aligned}
\left|\operatorname{atan}\left(\frac{y_{i}-y_{j}}{x_{i}-x_{j}}\right)-\operatorname{atan}\left(\frac{\left(y_{i}+\delta y_{i k}\right)-\left(y_{j}+\delta y_{j k}\right)}{\left(x_{i}+\delta x_{i k}\right)-\left(x_{j}+\delta x_{j k}\right)}\right)\right|<\left|\frac{\partial \mathbf{u}}{\partial x}\right| \Delta t, & i \neq j \quad(1)
\end{aligned}
$$

constrains the orientation of the sub-interrogation windows. Here $\left(x_{i}, y_{i}\right)$ and $\left(\delta x_{i k}, \delta y_{i k}\right)$ are the coordinate components of $x_{i}$ and $\delta x_{i k}$. This expression indicates that, since the maximum possible angle of rigid body rotation must be less than that produced by the maximum velocity gradient, the orientation of any two sub-pattern centers (i.e. the direction of a line connecting any two sub-patterns) must remain within a specific region.

The third criterion, based on the assumption that the flow is highly incompressible, restricts the maximum deviation of the displacement vector of each sub-pattern. This is very similar to that used in conventional PIV in which neighboring data are compared to extract invalid data; however, it is performed within the interrogation windows for the sub-pattern PIV method. The criterion is

$$
\frac{\left|\delta x_{i k}-\delta x_{\text {median }}\right|}{D}<\left|\frac{\partial u}{\partial x}\right| \Delta t
$$

where $\delta x_{\text {median }}$ is the median value of a possible combination of sub-interrogation window displacement vectors.

After elimination of data by these three criteria, those (few) possible combinations of sub-pattern displacement vectors remaining are termed "candidate sets" of sub-pattern displacement vectors. A typical case study shows that less than 10 sets of an initial $\mathrm{m}^{n}$ sets of possible combinations pass the elimination procedure for $d / D=0.1$ and $|\partial u / \partial x| \Delta t=0.30$. The small squares in Fig. 10b show a candidate set of displacement vectors when relating each square to its associated small square (sub-interrogation window) in Fig. 10a. (Note that the parallelogram in Fig. 10b is the true R-PIP boundary.)

The next step includes an artificial image distortion followed by a cross-correlation integral applied to the full-sized interrogation window. The artificial image distortion is used to remove an approximation to the first-order distortion caused by the velocity gradient that can not be accounted for using PIV techniques. A velocity-gradient tensor is determined for each candidate set by finding the least squares solution of a combination of two linear systems. The first system is

$$
\left[\begin{array}{ll}
x_{1} & y_{1} \\
x_{2} & y_{2} \\
\cdots & \cdots \\
x_{m} & y_{m}
\end{array}\right]\left[\begin{array}{ll}
\chi_{1} & \chi_{3} \\
\chi_{2} & \chi_{4}
\end{array}\right]=\left[\begin{array}{cc}
\delta x_{1 j} & \delta y_{1 j} \\
\delta x_{2 j} & \delta y_{2 j} \\
\cdots & \cdots \\
\delta x_{m j} & \delta y_{m j}
\end{array}\right]
$$

The velocity-gradient tensor can be determined using the solution of Eq. (13), and the equation

$$
\left[\begin{array}{ll}
\frac{\partial u}{\partial x} & \frac{\partial u}{\partial y} \\
\frac{\partial v}{\partial x} & \frac{\partial v}{\partial y}
\end{array}\right]=\left[\begin{array}{ll}
\chi_{1} & \chi_{2} \\
\chi_{3} & \chi_{4}
\end{array}\right] \frac{1}{\Delta t}
$$

An artificial, locally linear distortion (first-order approximation) is applied to the (full-sized) I-PIP. That is, every point of a (new) distorted I-PIP is mapped from the original (nondistorted) I-PIP, the relation between them is

$\left[\begin{array}{l}x \\ y\end{array}\right]_{\text {distored }}=\left[\begin{array}{cc}1+\frac{\partial u}{\partial x} & \frac{\partial u}{\partial y} \\ \frac{\partial v}{\partial x} & 1+\frac{\partial v}{\partial y}\end{array}\right]\left[\begin{array}{l}x \\ y\end{array}\right]_{\text {non-distored }}$

The distorted I-PIP is produced by transferring the intensity value at an arbitrary point $(x, y)_{\text {non-distored }}$ of the original image to its new, distorted position, $(x, y)_{\text {distored }}$. Since the original image intensity function is defined on discrete grid points, but the vector $(x, y)_{\text {distored }}$ may have between-grid-point values, a surface-fitting technique is required to assign those grid points with (interpolated) intensity values. Since there exist points that may cross the borders of the I-PIP, the $(x, y)_{\text {non-distored }}$ in practice require all points available, namely the entire S-PIP. The particle image presented in Fig. 10 is a distorted I-PIP; note the elongation/distortion of the originally circular spots.

A cross-correlation function is calculated from each distorted (full-sized) I-PIP (i.e. from each candidate set a distorted I-PIP is generated) and the S-PIP; however, there exists only one global cross-correlation-function maximum from amongst the candidate sets. Since the I-PIP has been distorted, the signal decay caused by the velocity gradient is eliminated if an appropriate distortion has been applied (i.e. Eq. (6) is satisfied for the full-sized, properly distorted I-PIP) such that the target cross-correlation coefficient is distinguished easily from the noise. It is a reasonable assumption that the largest crosscorrelation coefficient is produced by the artificial distortion closest to the actual (true) value, and it is thus chosen as the target. Therefore, after these manipulations, the target displacement of the particle image pattern is determined, even when Eq. (6) is not satisfied with the (un-distorted) fullsized I-PIP. Fig. 10f shows a cross-correlation integral of the full-sized, distorted I-PIP (Fig. 10d) and the original, nondistorted S-PIP. A very clear (distinguishable) signal emerges, and it is nearly coincident with the true target displacement vector.

Further iterations can be conducted and a more accurate estimation may be achieved. A similar procedure of choosing effective sub-patterns (small squares in Fig. 10d), performing cross-correlation integration between sub-patterns and the S-PIP, examining possible combinations of sub-pattern displacements (small squares in Fig. 10e), and then the distortion step, may be performed iteratively.

Figure 11 shows such a series of tests with 5 iterations. More than three-hundred tests are conducted for each condition. The cross-correlation coefficients are presented in the same manner as those in Fig. 9; however, the accuracy (indicated by the maximum error) is included in the upper six graphs within Fig. 11. The rectangles with larger horizontal width in the lower six graphs indicate the (true) signal cross-correlation coefficients (generated by correlated particles), while the noise maxima (as defined previously, are the cross-correlation peaks other than the true signal and are generated by non-related particles) are represented by narrower rectangles. For comparison purposes, the cross-correlation coefficients generated by conventional PIV are also included as the extreme 
left-hand-side data, noted as the 0th iteration - adjacent to the first iteration using Sub-pattern PIV. These figures clearly show that a distinguishable target (rather than noise) is achieved by using Sub-pattern PIV for all the situations tested, even when the velocity gradient is well-above the value suggested by Eq. (6). And, the accuracy is improved by at least one order. (Note that a similar claim is made for the PID method, but only if the interpolation step after the first iteration (a conventional PIV estimate) does not give an incorrect prediction that causes divergence in the later iterations.) It is mentioned that the improvement in accuracy is applicable for both the estimations of full-sized and sub-pattern interrogation windows. The maximum error, presented in the uppermost six graphs in Fig. 11, is not affected by a higher velocity gradient; however, a higher percentage of correlated particles does result in a more accurate velocity estimation. The cross-correlation coefficients and the accuracy of estimations attain their maximum values after the second iteration in most cases, while the noise remains constant over all iterations.

To demonstrate the effectiveness of the Sub-pattern PIV technique and the Brewster-angle imaging in measuring a real flow, Fig. 12 is included. (For a more complete investigation of vorticity and velocity fields beneath gravity-capillary waves, the reader is referred to Lin and Perlin.) This figure shows the vorticity distribution beneath a $6.70 \mathrm{~Hz}$ gravity-capillary wave with a steepness of 0.21 . In the figure, the progressive wave is presented with its forward face on the right, and hence parasitic capillaries are seen on the forward (and to a lesser extent on the leeward) face with mostly clockwise (defined
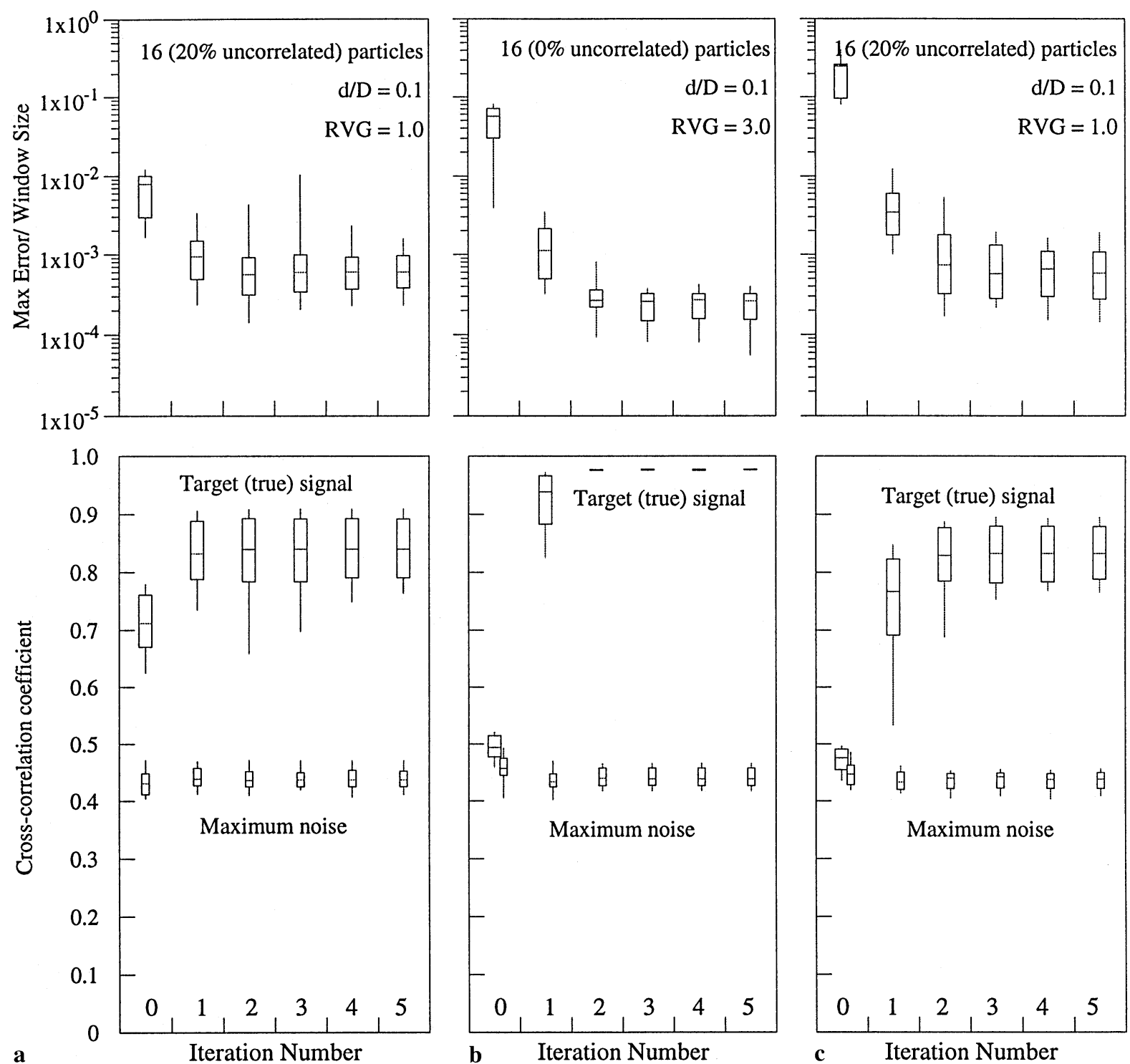

Fig. 11a-f. The accuracy and validity improvement by using Sub-pattern PIV. The relative velocity gradient (RVG) is defined as $(\partial u / \partial y) \Delta t(D / d)$; it has a value of one when Eq. (6) is exact. The upper and lower ends of the rectangular boxes represent the 75th and 25 th percentile of the sorted cross-correlation coefficients, while the median value is presented as the vertical line within the rectangle. The tail lines on each side of the boxes show the 95th and 5th percentiles, respectively 

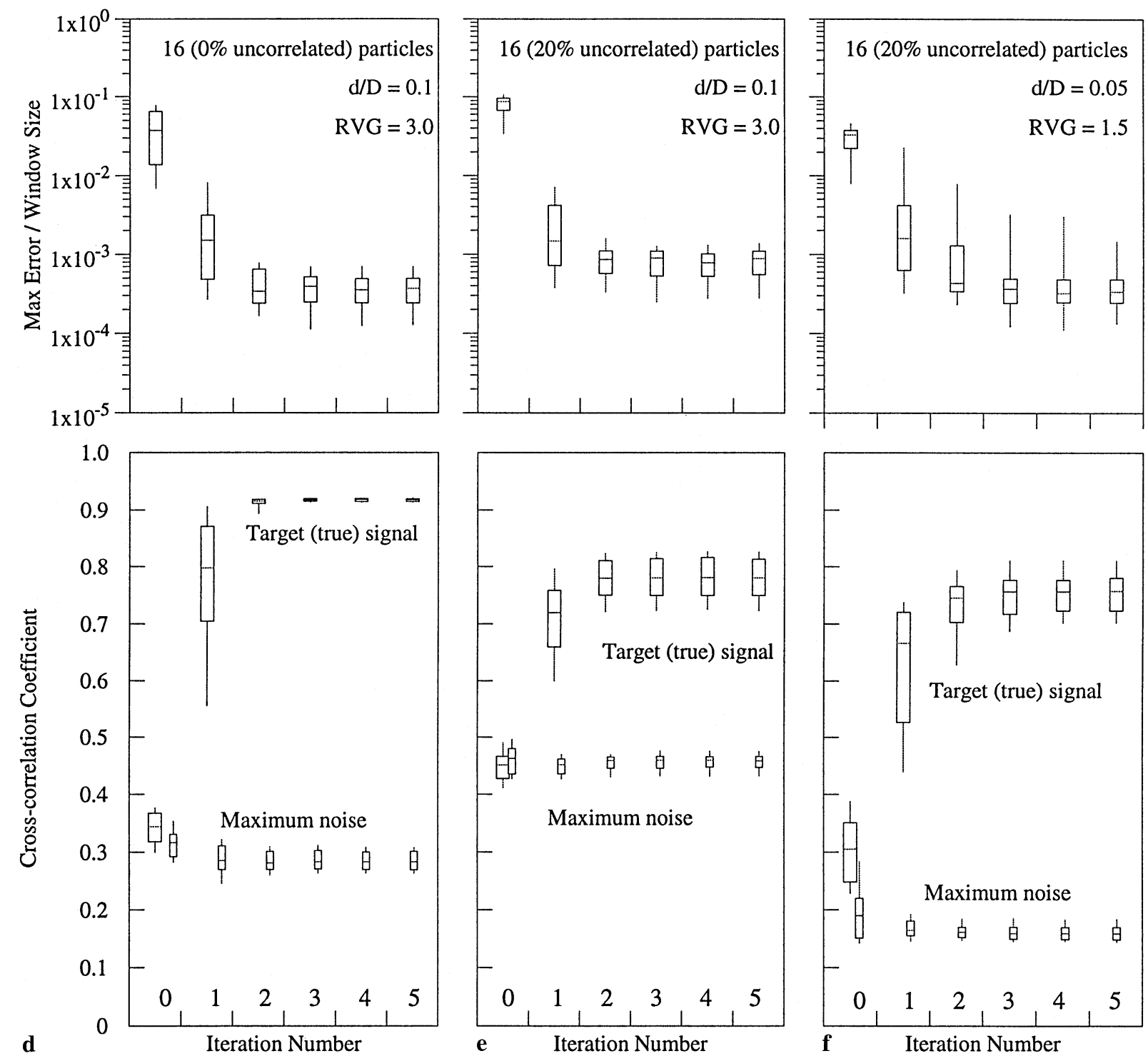

Fig. 11. Continued

$6.70 \mathrm{~Hz}$, wavelength $=50.0 \mathrm{~mm}, \mathrm{ka}=0.16$

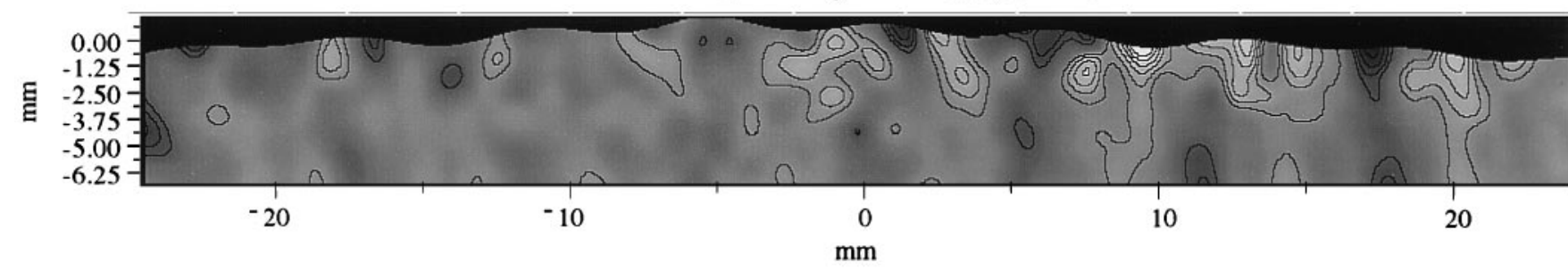

$\begin{array}{lllllllll}-40 & -30 & -20 & -10 & 0 & 10 & 20 & 30 & 40\end{array}$

Vorticity (1/s) Contour lines@ ( $+/-) 10,15,20,25,30,35$

Fig. 12. Vorticity distribution beneath a $6.70 \mathrm{~Hz}$ gravity-capillary wave with steepness, ka, 0.21 . Axes are in $\mathrm{mm}$ (Lin and Perlin). 
positive) vorticity immediately upstream of the capillary troughs. The capillary crests exhibit counterclockwise (defined negative) vorticity. The sign of the vorticity in the vicinity of the capillaries is in general agreement with theory and expectation.

\section{7}

\section{Discussion and concluding remarks}

The Brewster-angle viewing technique, a schematic of which is presented in Fig. 1c, successfully removes undesirable surfacereflected light that would otherwise appear, in a horizontallyviewed image that includes the air-water interface, as particles above the (undetectable) free surface. As shown in Fig. 3b, the method is very effective and in repeated application proved to be very robust.

By using Sub-pattern PIV, the restriction that large velocity gradients be disallowed is relaxed (as compared to conventional PIV). It is not removed completely, but relaxed significantly. Obviously, the Sub-pattern PIV procedure increases processing time. In the worst situation, it is $m k$ times slower than conventional PIV for each iteration, while an analysis for average examples shows a factor of 10 increase in CPU time. Recall that $m$ is the number of sub-patterns in the interrogation window, and $k$ is the number of possible displacement candidates for which the cross-correlation integral must be performed. Since the fast Fourier transform (FFT) technique is applied usually to calculate the crosscorrelation integration, the smaller sub-pattern window may decrease (step-down) the matrix size. For example, a $32 \times 32$ pixel, full-sized interrogation window with 10 pixels of searching range requires a $64 \times 64$ pixel matrix to apply the FFT analysis. The $10 \times 10$ pixel sub-pattern windows with 10 pixels of searching range require only a matrix of size $32 \times 32$ pixels. Therefore, only $0.17 k$ of additional CPU time is required for each iteration under this window size arrangement.

Since the Sub-pattern PIV is most effective and advantageous for the high velocity-gradient portion of the flow, elsewhere, conventional PIV should be used for the velocity estimate. After using conventional PIV, as problems arise in high vorticity regions (i.e. a poor signal/noise ratio is present or spurious estimates are found when comparing the vectors with its neighboring estimates), Sub-pattern PIV should be applied to overcome the velocity-gradient effects. Thus, the processing time consumed by the Sub-pattern PIV method can be minimized.

The velocity estimates of sub-pattern interrogation windows provide higher spatial resolution and accuracy for the entire flow field, and they can interface with the Sub-pattern PIV. (Recall that the displacements of sub-patterns used for estimating the appropriate artificial distortion are ready for use as high-resolution estimates.) Moreover, the information provided by sub-patterns to determine the appropriate artificial distortion is essentially equivalent to the final vorticity (as defined as the velocity differential) results achieved with (high-resolution) sub-patterns.

\section{References}

Adrian RJ (1991) Particle-imaging techniques for experimental fluid mechanics. Ann Rev Fluid Mech 23: 261-304

Boillot A; Prasad AK (1996) Optimization procedure for pulse separation in cross-correlation PIV. Exp Fluids 21: 87-93

Huang HT; Fiedler HE; Wang JJ (1993a) Limitation and improvement of PIV. Part I: Limitation of conventional techniques due to deformation of particle image patterns. Exp Fluids 15: 168-174

Huang HT; Fiedler HE; Wang JJ (1993b) Limitation and improvement of PIV. Part II: Particle image distortion, a novel technique. Exp Fluids 15: 263-273

Keane RD; Adrian RJ (1990) Optimization of particle image velocimeters. Part I: Double pulsed system. Meas Sci Technol 1: 1202-1215

Keane RD; Adrian RJ; Zhang Y (1995) Super-resolution particle imaging velocimetry. Meas Sci Technol 6: 754-768

Lin HJ (1997) The vortical structure of capillary-gravity waves: an experimental investigation. Ph.D. Dissertation, University of Michigan

Lin HJ; Perlin M (1998) The velocity and vorticity fields beneath gravity-capillary waves exhibiting parasitic ripples, Phys Fluids, sub judice

Perkins RJ; Hunt JCR (1989) Particle tracking in turbulent flows. Adv Turbulence 2: 289-291

Westerweel J (1994) Efficient detection of spurious vectors in particle image velocimetry data. Exp Fluids 16: 236-247

Willert CE; Gharib M (1991) Digital particle image velocimetry. Exp Fluids 10: 181-193 